\title{
Acute Respiratory Distress Syndrome (ARDS) Case Report after a Caesarean Section Related to the Immune Reconstitution Inflammatory Syndrome
}

\author{
Álvaro Ramiro Ruiz, Pilar Reboto Cortés, Ignacio García Sánchez, Juan Emilio Losa, \\ Miguel Miró Murillo, Santiago García del Valle y Manzano \\ Hospital Universitario Fundación Alcorcón, Madrid, Spain \\ Email: aramiro@fhalcorcon.es, PReboto@fhalcorcon.es, jigarcias@fhalcorcon.es, jelosa@fhalcorcon.es, \\ mmiro@fhalcorcon.es, sgarcia@fhalcorcon.es
}

Received 26 May 2014; revised 21 July 2014; accepted 8 August 2014

Copyright (C) 2014 by authors and Scientific Research Publishing Inc. This work is licensed under the Creative Commons Attribution International License (CC BY). http://creativecommons.org/licenses/by/4.0/ (c) (7) Open Access

\begin{abstract}
During pregnancy, a cellular immunosuppressant status is produced. It is characterized by anti-inflammatory cellular responses that allow embryonic implantation. The reversal of these changes during the postpartum period may result in overt clinical manifestations of otherwise quiescent or latent infections. We present an Acute Respiratory Distress Syndrome (ARDS) case after a caesarean section related to the Immune Reconstitution Inflammatory Syndrome (IRIS) in a Guinean black woman. The patient was treated with large doses of corticosteroids showing a great clinical and radiological improvement.
\end{abstract}

\section{Keywords}

Immune Reconstitution Inflammatory Syndrome, Mycobacterium Tuberculosis, Pregnancy

\section{Introduction}

During pregnancy, a cellular immunosuppressant status is produced. It is characterized by anti-inflammatory cellular responses that allow embryonic implantation. The reversal of these changes during the postpartum period may result in overt clinical manifestations of otherwise quiescent or latent infections. We think it is possible, that the following case of Acute Respiratory Distress Syndrome (ARDS) after caesarean section could be explained throughout this pathophysiological basis.

How to cite this paper: Ramiro, Á., Cortés, P.R., Sánchez, I.G., Losa, J.E., Murillo, M.M. and del Valle y Manzano, S.G. (2014) Acute Respiratory Distress Syndrome (ARDS) Case Report after a Caesarean Section Related to the Immune Reconstitution Inflammatory Syndrome. Open Journal of Anesthesiology, 4, 197-201. http://dx.doi.org/10.4236/ojanes.2014.48028 


\section{Case Report}

A 33-years-old pregnant black woman from Guinea was admitted to the Emergency Obstetric Service. Her medical history reported that she suffered malaria and poliomyelitis during her childhood. She had been evaluated by a Dermatologist since 2005 because of nodular injuries in the calf of both legs. A skin biopsy was done and the result was lobular panniculitis without vasculitis. Her pregnancy was controlled in Spain. Since the $17^{\text {th }}$ week of pregnancy, she suffered abdominal pain and dysuria. The urine culture results were positive and Citrobacter koseri was isolated. She received an antibiotic treatment with fosfomycin. A blood test showed the enhancement of neutrophylia and reactive protein C (RPC). LDH 338 U/L; ALT 105 U/L; AST 140 U/L. During the second term of pregnancy, liver transaminases were in their normal range again. At this moment, the nodular skin injuries got worse and the histological study was repeated. The tuberculin skin test was done, but she did not come back to check the results.

Finally, at the $37^{\text {th }}$ week of pregnancy, she arrived to the Emergency room with bad general condition, high fever, exertional dyspnoea and no fetal perceptible movement. Her vital signs were monitored: Blood Pressure (BP) 110/77; Temperature (T) $37.6^{\circ} \mathrm{C}$; Heart Rate (HT) 122 bpm; pulsioximetry saturation without oxygen: 93\%. Breath Rate (BR) 26 bpm. An electrocardiogram was done showing sinus rhythm; 74 bpm. Neither jaundice nor acholic faeces were perceived. An urgent blood test was ordered whose results were the followings: Neutrophyls (89.30\%); RPC 40.80 and procalcitonin $0.35 \mathrm{ng} / \mathrm{ml}$. Total bilirrubin $2.70 \mathrm{mg} / \mathrm{dl}$; direct bilirrubin $2.60 \mathrm{mg} / \mathrm{dl}$; LDH $468 \mathrm{U} / \mathrm{L}$; ALT $231 \mathrm{U} / \mathrm{L}$; AST $513 \mathrm{U} / \mathrm{L}$. The results of the clotting study were: ttPA $51.60 \mathrm{~s}$; dimer D $12,941 \mathrm{ng} / \mathrm{ml}$. It was found Proteinuria in the Urine strip test $(70 \mathrm{mg} / \mathrm{dl})$. We suspected complicated urine tract infection, so we decided to order both urine and blood cultures before beginning a wide spectrum antibiotherapy with Ertapenem. Gynaecologist decided to induce labour, but 18 hours later, a pathological cardiotocographic trace was observed associated to severe fetal bradycardia. An emergency caesarean section under General Anaesthesia was performed. A living hypotonic female baby was born.

The patient was admitted to the Post-operative Care Unit extubated and haemodynamically stable without vasoactive drugs needed. She stayed in the Unit with progressive dysnoea and oliguria. She was tachypneic and bedridden despite of non invasive ventilation and volume reposition and diuretics. We observed bilateral whitecottony infiltrates on the X-ray examination that suggested an Acute Respiratory Distress Syndrome (ARDS) (Figure 1). We ordered an arterial blood gas test with a fraction of inspired oxygen $\left(\mathrm{FIO}_{2}\right)$ of $50 \%$ that showed: pH 7.42; $\mathrm{PaCO}_{2} 29.70 \mathrm{mmHg} ; \mathrm{PaO}_{2} 66.50 \mathrm{mmHg}$. Finally, the patient needed to be intubated and connected to protective mechanical ventilation.

We performed a Fibreoptic bronchoscopy. Bronchoalveolar lavage was positive for Mycobacterium Tuberculosis (MT), so the patient begun to receive an antituberculosis chemotherapy with no hepatotoxic drugs (Ethambutol, Levofloxacin, Pyrazinamide and Streptomycin). We maintained a wide spectrum antibiotherapy with Meropenem and two milligrams per kilogram of body weight of methylprednisolone were administrated.

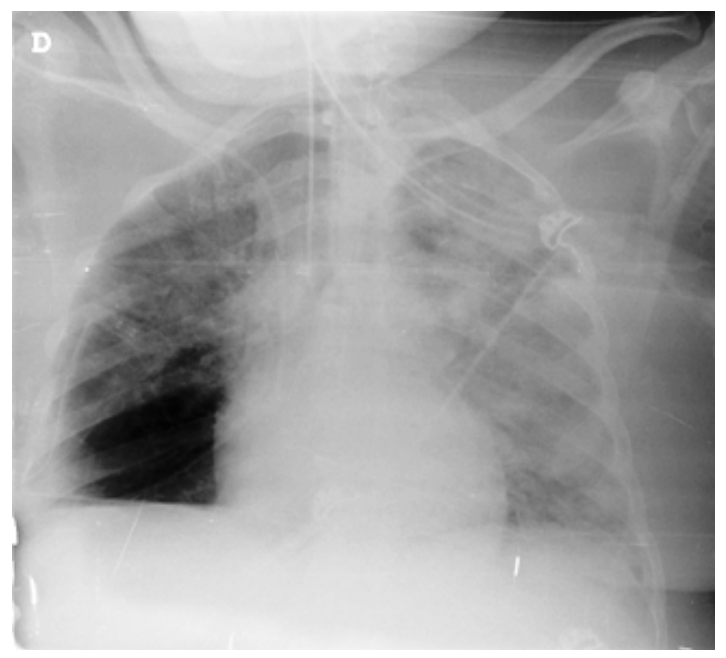

Figure 1. X-ray showing bilateral cotton infiltrates suggesting Acute Respiratory Distress Syndrome. 
The liver transaminases levels got higher: Total bilirrubin $3.30 \mathrm{mg} / \mathrm{dl}$; direct bilirrubin $3.20 \mathrm{mg} / \mathrm{dl}$; LDH 460 U/L; GPT 239 U/L; GOT 560 U/L. The results of the clotting study were: PT 15.72 seconds (9.0 - 13.0), PA 54.11\% (80.0 - 120.0), I.N.R. 1.4 (0.0 - 1.15), TTPA 58.03 secs. An abdominal echography was performed showing images according to chronic liver disease and portal hypertension. Both hepatotropic and Human Immunodeficiency virus (HIV) serologies were negative. The protein chain reaction test for Cytomegalovirus (CMV) was also negative. Other possible causes of chronic liver disease such as Autoimmune Hepatitis, Hemochromatosis, Wilson and alpha 1 anti tryptase deficiency were included in the differential diagnosis. The entire specific tests were negative.

Five days later, the liver transaminases decreased. Streptomycin and Levofloxacin were replaced by Isoniazid and Rifampicin. Her blood clotting function was re-established, so we performed a liver biopsy that showed noncaseating granulomas. Because of this finding, we ordered an Angiotensin-converting Enzyme (ACE) test. ACE had enhanced; $173 \mathrm{U} / \mathrm{L}$ (8 - $52 \mathrm{U} / \mathrm{L}$ ) leading to a possible diagnosis of Sarcoidosis. In the Computerized Tomography Scan (CT) there was no evidence of mediastinal lymph nodes (Figure 2).

The patient suffered a worsening of her respiratory status. She required protective mechanical ventilation with an increasing need of higher oxygen inspiratory fraction, multiple recruitment manoeuvres and prone ventilation position sessions. Finally, we performed a percutaneous tracheostomy. Due to a critical, life-threatening situation, we administrated high doses of methylprednisolone (1000 mg per day) for a week.

Seven days later, a clinical, radiological and analytic improvement appeared (Figure 3). The $\mathrm{PaO}_{2} / \mathrm{FiO}_{2}(\mathrm{P} / \mathrm{F}$

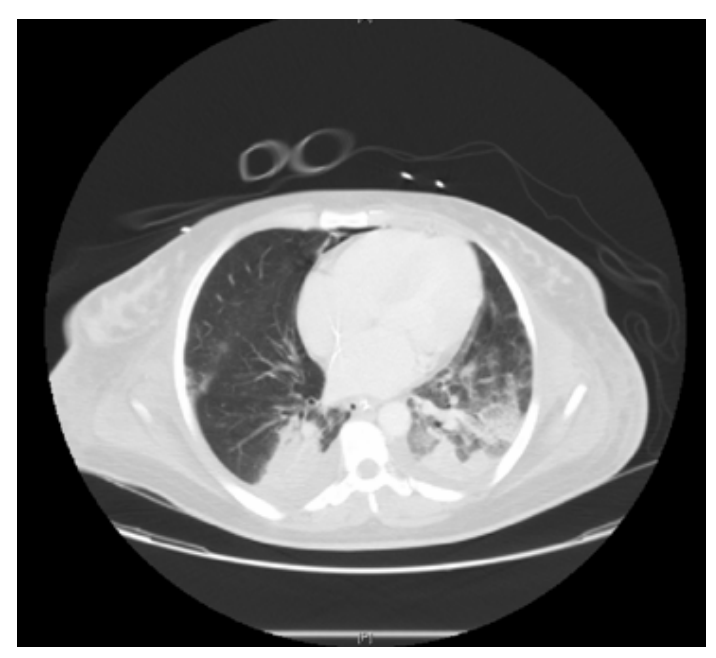

Figure 2. Computerized tomography not showing mediastinal lymph nodes.

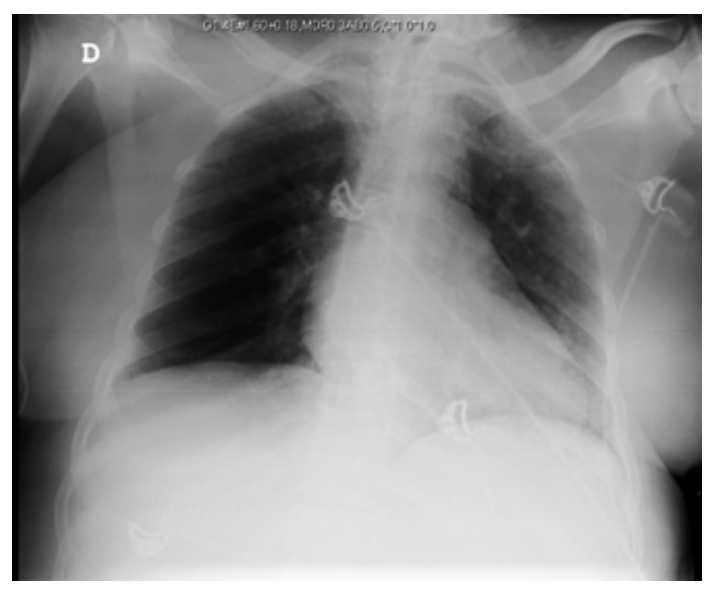

Figure 3. X-ray seven days after treatment with high doses of methylprednisolone. 
ratio) was greater than $300\left(\mathrm{PO}_{2} 168 \mathrm{mmHg}\right.$ with a $\mathrm{FIO}_{2}$ of 0.5$)$. Lastly, after a very slow ventilator weaning, the patient was discharged from the Post-operative Care Unit at her $35^{\text {th }}$ day of admission. A month later, she was discharged after suffering a tracheotomy wound infection by Pseudomona aerogynose as the only relevant complication.

\section{Discussion}

During pregnancy, a cellular immunosuppressant status is produced. It is characterized by anti-inflammatory cellular responses that allow embryonic implantation. The reversal of these changes during the postpartum period may result in overt clinical manifestations of otherwise quiescent or latent infections [1]. The Immune Reconstitution Syndrome (IRIS) has been well described in literature after the beginning of the anti-retroviral treatment in HIV infection cases. Meinjes et al. published a consensus in 2008 based on a standardized case definition of IRIS associated to Tuberculosis in this kind of patients [2]. This paper includes clear criteria for diagnosis but they cannot be extrapolated to another immune modulation circumstances such as pregnancy, due to the lack of publications on this subject. One of the TB-IRIS risk factors consists of suffering a subclinical infection at the beginning of the Highly Active Antiretroviral Therapy (HAART) and a very earlier beginning of the program [3]. As it happens in the immediate puerperium period; an increase of T helper 1 (Th1) over Th2 lymphocytes levels after HAART has been described. In fact, a MT specific lymphocyte population has risen in this situation [1]. For this reason, we think it is possible that this case of ARDS after a caesarean section could be explained throughout this pathophysiological basis. Lungs are the main organs affected by TB-IRIS and it can develop a respiratory failure [3]. The clinical scene consisted of a systemic pathology, overall with lung affection and liver granulomas so that we included Sarcoidosis as a diagnosis possibility. As far as we know, the disease aetiology is not completely known though it has been linked to several infectious agents such as MT. Therefore, mycobacterium genetic material has been isolated from granulomas in these patients. So that, distinguishing between Sarcoidosis and Tuberculosis infection can be exceptionally hard. In fact, both of them can coexist [4]. That is the reason why an ACE determination was included among the diagnosis test; $173.3 \mathrm{U} / \mathrm{L}$ (8.0 52.0). An ACE enhancement is found in 30 - 80 per cent of the cases, although false positive results have been found in 10 per cent of the patients suffering lung disease [5]. We did not find mediastinal lymph nodes in the CT scan. Nevertheless, in the third stage, the disease is accompanied by lung infiltrates without hiliar adenopathies [6]. Several cases of Sarcoidosis developing or recurring in HIV patients have been described, usually occurring after about 12 months of therapy [3]. Pregnancy has been said to ameliorate Sarcoidosis, probably of an immunecomprommised state [7]. In fact, it is uncommon to find a radiological worsening [8].

The finding of non caseating granulomas is not exclusive of Sarcoidosis. In a study consisting of 150 cases of adult tuberculosis; granulomas in liver biopsy were caseating in 58.7\%; noncaseating in $23.8 \%$ and atypical in $17.5 \%$ cases [9].

The absence of treatment response together with an antituberculosis chemotherapy and a clinical course, and the development of lung cotton infiltrates and a P/F ratio lower than 200 made us realize that an active tuberculosis infection was not the only problem. An immune mechanism as the core of the matter was considered as the main cause.

According to the corticosteroid systemic therapy, patients with Sarcoidosis in the second and third stages can show a radiological improvement in intersticial lung injuries. There is poor evidence about improvement in lung function [10]. The recommended daily dose of prednisone is $20-40 \mathrm{mg}$.

In case of a life-threatening situation in the context of respiratory failure due to TB-IRIS, corticosteroids are the mainstay of therapy [3]. The dose and the duration of the treatment are not well established [2]. Meintjes et al. assure in a randomized trial that $1.5 \mathrm{mg} \cdot \mathrm{kg}^{-1}$ day of prednisone during 2 - 4 weeks followed by $0.75 \mathrm{mg} \cdot \mathrm{kg}^{-1}$ for 2 weeks; reduces the need of hospitalization with no significant adverse effects as infections. In this case, 15 $\mathrm{mg} \cdot \mathrm{kg}^{-1}$ of methylprednisolone was not enough to achieve a meaningful clinical improvement. Finally, higher doses were necessary (1000 milligrams per day). A week later, the P/F ratio was higher than 450 .

\section{Conclussions}

Any immunomodulation circumstance worsens an underlying subclinic infection not only at the beginning of HAART in HIV positive patients.

To distinguish between Sarcoidosis and Tuberculosis infection can be exceptionally hard. Both of them can 
improve through the immunosuppressive effect which is produced by the corticosteroid therapy.

Although the corticosteroid indication in case of life-threatening situation is clear, there is no consensus about which active substance or which doses are better. In fact, we wonder why the reported dose in the literature was not enough in this case.

\section{References}

[1] Singh, N. and Perfect, J.R. (2007) Immune Reconstitution Syndrome and Exacerbation of Infections after Pregnancy. Clinical Infectious Diseases, 45, 1192-1199. http://dx.doi.org/10.1086/522182

[2] Meintjes, G., et al. (2008) International Network for the Study of HIV-Associated IRIS. Tuberculosis-Associated Immune Reconstitution Inflammatory Syndrome: Case Definitions for Use in Resource-Limited Settings. The Lancet Infectious Diseases, 8, 516-523. http://dx.doi.org/10.1016/S1473-3099(08)70184-1

[3] Meintjes, G., et al. (2010) Randomized Placebo-Controlled Trial of Prednisone for paradoxical Tuberculosis-Associated Immune Reconstitution Inflammatory Syndrome. AIDS, 24, 2381-2390.

[4] Agarwal, R. and Gupta, D. (2009) Tuberculous Sarcoidosis: Is It a Separate Entity? Lung India, 26, 61-62. http://dx.doi.org/10.4103/0970-2113.53225

[5] Baudin, B. (2005) [Angiotensin I-Converting Enzyme (ACE) for Sarcoidosis Diagnosis]. Pathologie Biologie (Paris), 53, 183-188. http://dx.doi.org/10.1016/j.patbio.2004.09.003

[6] Lynch III, J.P., Kazerooni, E.A. and Gay S.E. (1997) Pulmonary Sarcoidosis. Clinics in Chest Medicine, 18, 755-785.

[7] Ellafi, M. and Valeyre, D. (1999) Sarcoidosis and Pregnancy. Revue de Pneumologie Clinique, 55, 335-337. http://dx.doi.org/10.1016/S0272-5231(05)70417-2

[8] Amarapurkar, A1. and Agrawal, V. (2006) Liver Involvement in Tuberculosis-An Autopsy Study. Trop Gastroenterol, 27, 69-74.

[9] Paramothayan, N.S., Lasserson, T.J. and Jones, P.W. (2005) Corticosteroids for Pulmonary Sarcoidosis. Cochrane Database of Systematic Reviews, 18, Article ID: CD001114. http://dx.doi.org/10.1002/14651858.CD001114.pub2

[10] Narita, M., Yamada, S., Kikuta, H. and Togashi, T. (2000) Reconstitution of Humoral Immunity During Pregnancy. American Journal of Reproductive Immunology, 44, 148-152. http://dx.doi.org/10.1111/j.8755-8920.2000.440304.x 
Scientific Research Publishing (SCIRP) is one of the largest Open Access journal publishers. It is currently publishing more than 200 open access, online, peer-reviewed journals covering a wide range of academic disciplines. SCIRP serves the worldwide academic communities and contributes to the progress and application of science with its publication.

Other selected journals from SCIRP are listed as below. Submit your manuscript to us via either submit@scirp.org or Online Submission Portal.
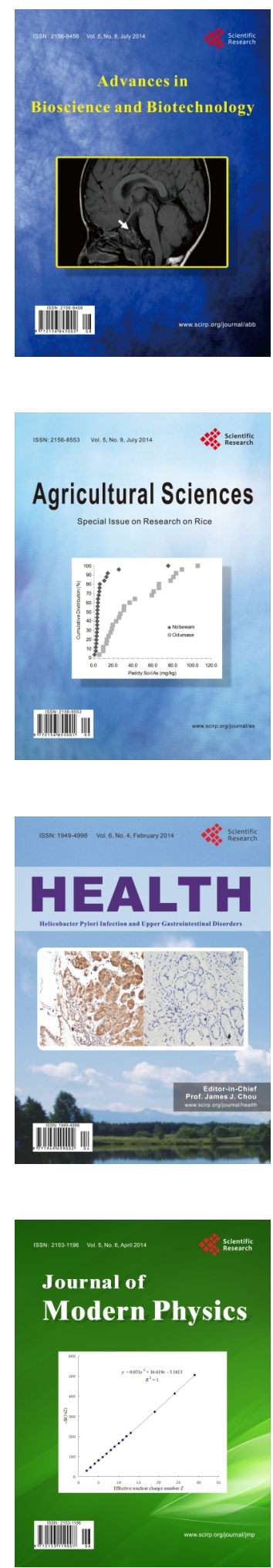
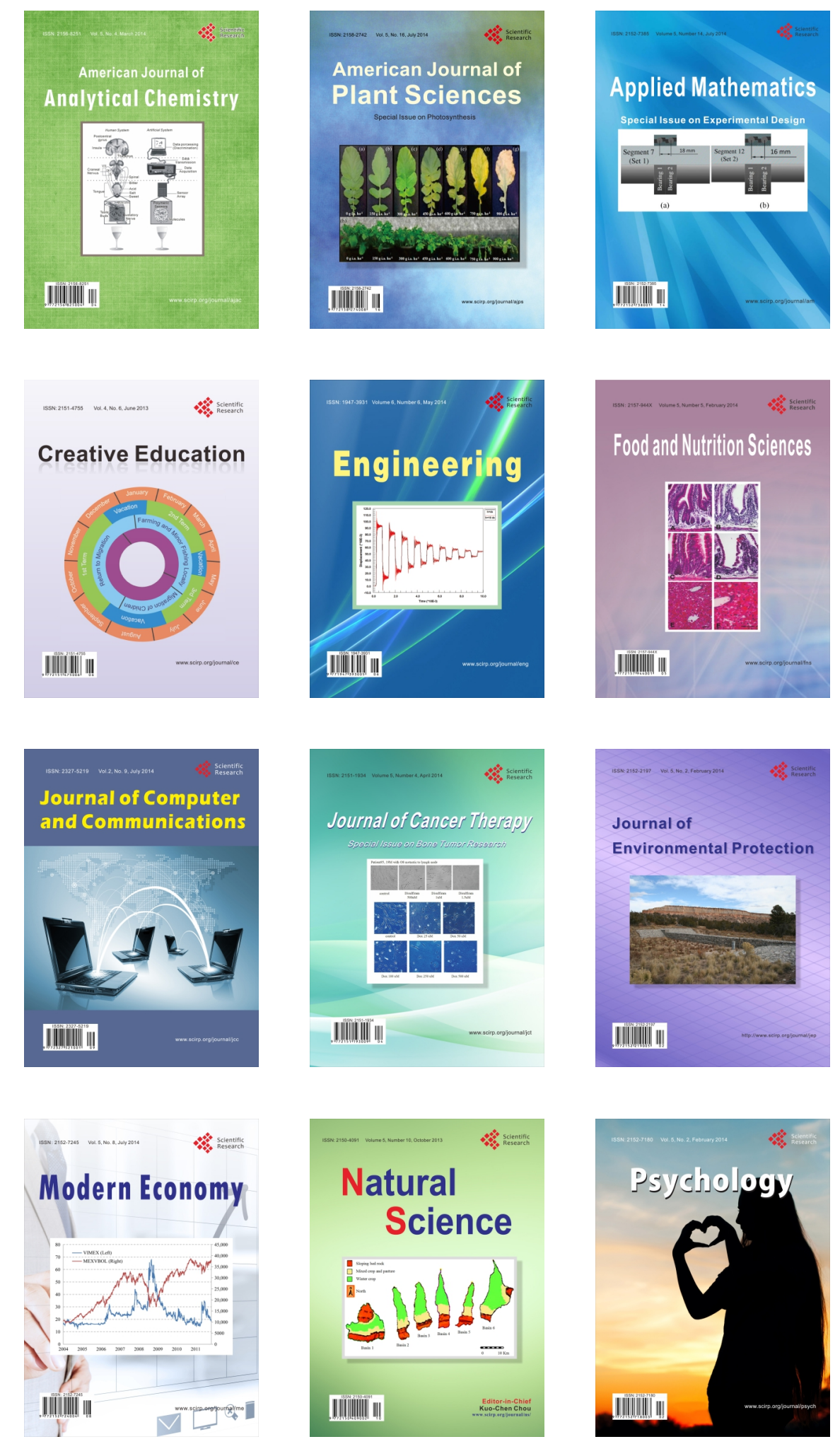